FORMATION Formation emploi

Revue française de sciences sociales

108 | octobre-décembre 2009

Les processus de professionnalisation

\title{
L'avenir appartient à ceux qui savent anticiper
}

Présentation de l'ouvrage

La Prospective des Métiers

Jean-Paul Cadet

\section{CpenEdition}

1 Journals

Édition électronique

URL : http://journals.openedition.org/formationemploi/2126

DOI : 10.4000/formationemploi.2126

ISSN : 2107-0946

Éditeur

La Documentation française

Édition imprimée

Date de publication : 1 décembre 2009

Pagination : 115-119

ISSN : 0759-6340

\section{Référence électronique}

Jean-Paul Cadet, "L'avenir appartient à ceux qui savent anticiper », Formation emploi [En ligne], 108 | octobre-décembre 2009, mis en ligne le 27 novembre 2009, consulté le 30 octobre 2020. URL : http:// journals.openedition.org/formationemploi/2126; DOI : https://doi.org/10.4000/formationemploi.2126

(c) Tous droits réservés 


\title{
Note de lecture
}

\section{L'Avenir appartient à ceux qui savent anticiper. Présentation de l'ouvrage "La Prospective des Métiers»}

\author{
Par Jean-Paul Cadet ${ }^{\star}$
}

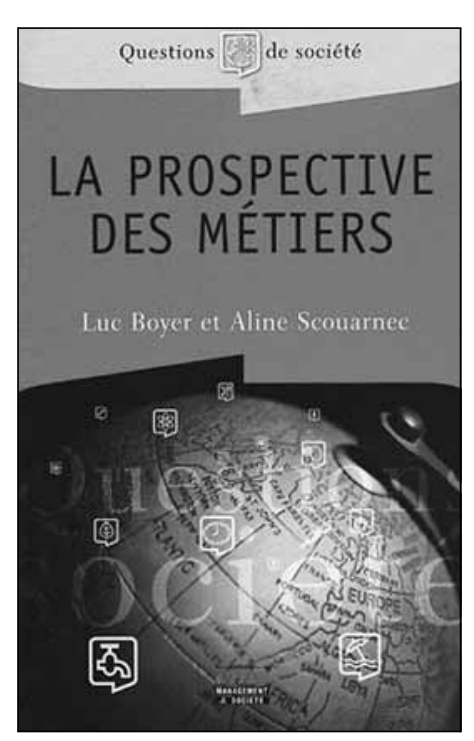

Il est de bon ton d'afficher une ambition prospective dans le champ de la relation formation-emploitravail. Depuis l'époque de la planification, l'État n'a cessé d'initier des travaux visant à prévoir, à anticiper ou à pronostiquer l'avenir quantitatif ou qualitatif des emplois pour tenter d'ajuster l'appareil de formation. Aujourd'hui, il perpétue pleinement cette tradition à travers notamment les travaux du Conseil d'analyse stratégique et de la Direction de l'animation de la recherche, des études et de la statistique du ministère de l'Emploi (CAS-Dares, 2005). Acteurs de plus en plus agissants dans le champ, les régions et les branches professionnelles entendent également connaître de mieux en mieux l'avenir des formations et des emplois par le biais de leurs observatoires : les Observatoires régionaux de l'emploi et de la formation
(OREF) pour les premières et les Observatoires prospectifs des métiers et des qualifications (OPMQ) pour les secondes. Les entreprises, et plus largement les organisations, se préoccupent elles-mêmes de plus en plus de prospective en matière de gestion des ressources humaines. La gestion prévisionnelle des emplois et des compétences (GPEC) est d'ailleurs devenue, après plusieurs années d'expérimentation et de tâtonnement, une pratique obligatoire pour les plus grandes d'entre elles. La nouvelle crise de l'emploi ne semble pas aujourd'hui entamer le mouvement. Bien au contraire, elle conduit à dénoncer le court-termisme et l'imprévoyance de certaines formes de gestion, et à explorer plus que jamais le futur en matière d'activités et de compétences, ne serait-ce que pour en sortir le mieux possible.

* Jean-Paul Cadet est chargé d'études en gestion des ressources humaines au Céreq, au sein du département Travail, Emploi, Professionnalisation. Il s'intéresse tout particulièrement aux processus de professionnalisation des salariés au sein des entreprises et des organisations publiques. II participe actuellement au pilotage d'une enquête sur les professions intermédiaires en entreprise. 
Mais voilà, si l'idée d'une prospective dans le domaine des emplois, des formations et du travail en général est sans aucun doute utile socialement, encore faut-il savoir en construire une qui se révèle suffisamment rigoureuse et légitime. En effet, comment appréhender et analyser de manière convaincante l'avenir, c'est-à-dire ce qui ne s'est pas encore produit? Et comment concevoir une prospective en mesure de se faire entendre, notamment par les acteurs sociaux à qui elle s'adresse au premier chef? Ces questions d'ordre méthodologique sont certainement des rengaines pour les prospectivistes. Elles n'en demeurent pas moins des questions impossibles à éluder et en tout cas irrésolues à ce jour.

L'ouvrage de Luc Boyer et d'Aline Scouarnec, $\mathrm{La}$ Prospective des Métiers, a sans conteste le mérite d'affronter, plutôt à son avantage, ces défis méthodologiques. Les auteurs préconisent en effet une approche qui se caractérise à la fois par une grande rigueur et un sens très opérationnel. Spécialistes en sciences de gestion, ils la destinent en priorité aux organisations qui s'interrogent sur le devenir de leurs activités et de leurs emplois, que ce soit directement par le biais de leur direction ou des autres parties prenantes (salariés, organisations syndicales...), ou plus indirectement via ces configurations inter-organisationnelles que forment les branches et les territoires. Intitulée tout simplement «Prospective des Métiers » (PM), leur méthode est présentée comme «une démarche d'anticipation des futurs possibles en termes de compétences, d'activités, de responsabilités d'un métier. Elle permet ainsi d'imaginer les possibles savoirs et qualifications, expertises ou savoir-faire professionnels, comportements et savoirêtre, qui seront demain les plus à même de servir l'individu et l'organisation. Elle nécessite pour cela une co-construction par les acteurs-experts du ou des métiers analysés du devenir possible de ce ou ces métiers. Elle englobe ainsi une réflexion sur le métier individuel et l'organisation du travail ».

Principal fondement conceptuel de cette approche, la vieille notion de métier - qui ne cesse décidément de faire son retour $!^{1}$ - présente l'avantage de lui conférer de la légitimité sociale. En effet, très ancrée

${ }^{1}$ Mireille Dadoy soulignait déjà ce retour il y a vingt ans (Dadoy, 1989). dans notre culture latine et nos imaginaires professionnels, elle reste parlante et valorisante pour nombre d'acteurs sociaux. Notamment, elle représente, pour les titulaires d'emploi, un repère identitaire qui leur offre la perspective de se référer à une continuité à la fois spatiale et temporelle (Piotet, 2002; Mahlaoui, 2008). Spatiale, car le métier renvoie à un espace qui dépasse le cadre strict de la situation de travail et de l'entreprise. Temporelle, car il invite à s'inscrire dans une histoire collective et personnelle, apte à relier passé, présent et avenir, sans rupture brutale et incontrôlée de trajectoire. En cela, comme les auteurs l'avancent, l'appui sur cette notion permet de dépasser les approches classiques de gestion par les compétences, lesquelles génèrent de la "désocialisation », en raison de l'extrême individualisation qu'elles induisent mais aussi, avonsnous envie d'ajouter, par la forte polarisation sur l'entreprise qu'elles entraînent.

La conception de la prospective développée au sein de l'ouvrage a également de quoi convaincre. Dans une perspective de long terme (entre cinq et dix ans au moins), cette prospective vise à repérer les multiples futurs possibles pour un métier ou une famille de métiers donnée, et à les traduire sous la forme de scénarios. Globale, elle entend embrasser tous les facteurs susceptibles d'influencer le devenir de ce ou ces métiers : l'évolution des stratégies d'entreprise, les mutations technologiques, économiques, juridiques, institutionnelles, sociologiques... Cette prospective rompt surtout avec la logique de la prévision et sa prétention à définir le ou les quelques scénarios à venir les plus probables sur la base d'hypothèses préconçues et de données quantitatives. Les auteurs appréhendent ainsi le temps dans toute sa dimension « humaine » et qualitative, en considérant sa liaison irréductible à des vécus d'acteurs, son hétérogénéité, son imprévisibilité, ses discontinuités. Ils conçoivent dès lors la prospective comme une démarche ayant vocation à aider les acteurs sociaux à prendre conscience des différents avenirs envisageables et à tenter de construire, dès à présent, celui qui leur semble, sinon préférable, du moins le plus raisonnable.

Cette conception de la prospective n'est pas nouvelle. Elle puise chez un auteur aussi ancien qu'illustre que Gaston Berger (Berger, 1964) et reprend tous les canons que l'on attribue désormais à 
la prospective, par opposition à la prévision et $a$ fortiori aux simples projections de tendances, lesquelles ont pu montrer par le passé leurs limites. Le renouveau apporté par Luc Boyer et Aline Scouarnec se situe davantage dans la mise en application de cette logique prospective au champ des métiers et au monde de l'organisation et du management. La façon d'appréhender l'avenir dans le cadre de la prospective des métiers est notamment en mesure d'enrichir les approches classiques et dominantes en matière de GPEC, trop souvent adossées à des logiques de prévision et de projection.

Sur un plan plus méthodologique, la PM présente un grand attrait dans le sens où elle s'inscrit parmi les méthodes dites «d'experts», dans le prolongement d'approches comme la méthode Delphi et à l'image de ce qui se fait de plus en plus aujourd'hui en matière de prospective-métiers, comme par exemple à l'heure actuelle au Céreq (Kalck, 2008). Les auteurs se fondent sur le principe d'une coconstruction de l'analyse entre les chercheurs et des « acteurs-experts» invités à s'impliquer. Ces derniers sont des professionnels qui exercent le métier analysé à un niveau d'expertise. «Un expert est une personne qui, de par ses expériences actuelles et passées, sa connaissance du métier et du milieu, ses compétences pointues, tant techniques que relationnelles, est le plus à même de pouvoir s'exprimer sur l'ensemble des possibles, les ruptures, les signaux faibles, etc., relatifs au système étudié dans l'exercice prospectif $\gg$. En ce sens, la méthode PM consiste avant tout à organiser un recueil de points de vue provenant d'acteurs-experts, avec l'intention de dégager et de mesurer leurs convergences comme leurs divergences. Pour ce faire, elle s'appuie concrètement sur une approche multiméthodes (analyse de l'état de l'art, entretiens, questionnaires, groupes de travail) soigneusement balisée en plusieurs étapes : "appréhension contextuelle», «pré-formalisation", " construction » (à l'occasion d'une journée de travail rassemblant l'ensemble des acteurs-experts ayant été sollicités), «validation 》 (à l'issue de cette journée de travail), "enquête en extension » (facultatif).

Présentés au sein de la seconde partie de l'ouvrage, les exemples d'application attestent amplement de la robustesse conceptuelle et méthodologique de la PM, de même que de sa grande souplesse, puisque ces exemples portent sur des univers très différents, aux enjeux extrêmement variés. Tantôt ils concernent un espace inter-organisationnelle (un territoire ou une famille professionnelle), tantôt une simple organisation. Concernant ces applications, il faut surtout souligner la qualité des résultats produits et plus particulièrement la portée heuristique de nombreux scénarios élaborés. Pour ne prendre qu'un seul exemple, celui de l'étude prospective ayant porté sur les métiers de la fonction ressources humaines, tout un ensemble de scénarios essentiels pour la compréhension de leur dynamique a pu être mis en évidence. Certains étaient sans doute prévisibles, comme le rôle croissant de l'informatique dans l'exercice de ces métiers, l'ouverture internationale accrue de la fonction ou la poursuite du développement de la gestion par les compétences au sein des firmes, avec ce qu'elle implique pour la professionnalité des gestionnaires en ressources humaines. D'autres scénarios apparaissaient moins prévisibles, telle la judiciarisation de la fonction ou encore son externalisation à grande échelle, non plus limitée de ce fait à la paye.

Cependant, force est d'admettre que la référence au métier peut paraître circonscrite dans cette approche. Pour les auteurs, et selon une représentation répandue aujourd'hui, le métier ne désigne que "l'ensemble des compétences individuelles requises pour accomplir une activité et occuper un poste ». À aucun moment, il n'est question de cet autre attribut traditionnel du métier que sont les règles éthiques de son exercice, alors même qu'une prospective aurait tout à gagner à questionner aussi leur devenir. En outre, les auteurs ne tirent peut-être pas suffisamment partie de la dimension collective attachée ordinairement au métier. Certes, leur méthode passe par l'organisation d'une journée de travail avec l'ensemble des acteurs-experts, créant ainsi les conditions d'une co-construction des scénarios. Mais cette polarisation sur une seule journée ne réduit-elle pas trop la part laissée à la dynamique collective et aux échanges entre pairs, étant entendu qu'ils pourraient constituer aussi le ferment d'une élaboration dépassant la simple confrontation des différents points de vue individuels ?

Au niveau méthodologique, le problème réside aussi dans l'appel exclusif fait à des titulaires d'emploi, 
considérés de surcroît comme des experts dans leur métier. Si elle a le grand mérite de redonner la parole à ceux qui exercent les métiers, cette façon de procéder pose la question du statut des discours ainsi collectés. Dans quelle mesure ne sont-ils pas de simples discours d'autopromotion ou de défense des métiers prenant trop peu de distance? Sont-ils d'ailleurs en capacité de diagnostiquer correctement toutes les évolutions à venir? Afin de limiter ces risques de biais inhérents à toute méthode fondée sur des dires d'acteurs, ne faudrait-il pas interroger aussi les autres acteurs susceptibles de peser à leur façon sur l'évolution de ces métiers (chefs d'entreprise, juristes, pouvoirs publics...), voire prendre le soin de confronter cette démarche qualitative à des protocoles d'enquête plus classiques, fondés sur une logique prévisionnelle? Le choix de se cantonner à des experts risque quant à lui de limiter le recueil de perceptions et de représentations aux professionnels les plus aguerris, en faisant fi de celles des plus jeunes ou des plus «originaux », peut-être révélatrices aujourd'hui des signaux annonciateurs des changements les plus importants pour demain. Ce choix est susceptible également d'uniformiser en partie les points de vue développés. Quand bien même les auteurs en appellent à une diversification des profils d'experts, ils débouchent de fait sur des résultats qui relèvent d'une certaine forme d'unanimisme. Enfin, se pose concrètement le problème du choix effectif des experts : comment les repérer concrètement et de manière suffisamment consensuelle?
La Prospective des Métiers de Luc Boyer et Aline Scournec n'en demeure pas moins une contribution très convaincante. Ces deux auteurs ont su habilement appliquer les canons de la prospective au champ des métiers, un domaine où les besoins d'éclairage sur les évolutions à venir restent immenses. Ils ont développé une méthode susceptible de se révéler utile au management stratégique des organisations et à la gestion des ressources humaines, mais aussi aux salariés euxmêmes, lesquels sont de plus en plus appelés à endosser le premier rôle dans la construction de leur parcours professionnel et donc à mieux connaître la dynamique des métiers et des compétences.

Certes, on pourra s'étonner de la vision très «marchande» de l'être humain au travail adoptée parfois par les auteurs, à l'encontre de leur thèse sur les risques de désocialisation des pratiques de gestion par les compétences; ils écrivent ainsi : "l’homme doit apprendre à s'évaluer et à se vendre comme un produit.» Mais on retiendra surtout la grande force pédagogique de leur ouvrage. Grâce à une présentation bien structurée et solidement argumentée, il semble pleinement apte à faire prendre conscience aux acteurs sociaux de l'importance de penser le futur des métiers pour construire efficacement l'action présente en matière d'emploi et de formation, tout en leur livrant de précieuses ficelles méthodologiques. En définitive, il s'impose comme une contribution à même d'aider les acteurs sociaux à savoir mieux anticiper l'avenir des métiers et à éviter d'être condamnés à devoir le subir en totalité.

\section{Références de l'ouvrage}

Boyer Luc et Scouarnec Aline, (2009), La Prospective des Métiers, Éditions Management \& Société, collection «Questions de société », 368 p. 


\section{Bibliographie}

Berger G. (1964), Phénoménologie du temps et prospective, PUF.

CAS-Dares (2005), Les Métiers en 2015, rapport.

Dadoy M. (1989), Le retour au métier, Revue française des affaires sociales, $\mathrm{n}^{\circ}$ 4, pp. 69-102.
Kalck P. (2008) « Une vision prospective des métiers développés par les professionnels du bâtiment», Bref-Céreq, $\mathrm{n}^{\circ} 254$, juillet.

Mahlaoui S. (avec la coll. de Cadet J.-P. et Rousseau M.) (2008), «Analyser les métiers pour gérer les mobilités en entreprise ", Bref-Céreq, n² 249, février.

Piotet F. (dir.) (2002), La révolution des métiers, PUF. 